\title{
An automated toolbox to predict single subject atrophy in presymptomatic Granulin mutation carriers
}

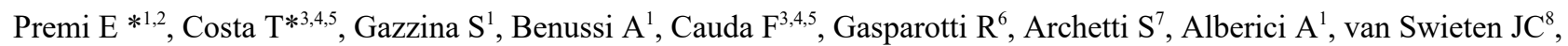
Sanchez-Valle $\mathrm{R}^{9}$, Moreno $\mathrm{F}^{10,11}$, Santana $\mathrm{I}^{12,13,14}$, Laforce $\mathrm{R}^{15}$, Ducharme $\mathrm{S}^{16,17}$, Graff $\mathrm{C}^{18,19}$, Galimberti $\mathrm{D}^{20,21}$, Masellis $\mathrm{M}^{22}$, Tartaglia $\mathrm{C}^{23}$, Rowe $\mathrm{JB}^{24}$, Finger $\mathrm{E}^{25}$, Tagliavini $\mathrm{F}^{26}$, de Mendonça $\mathrm{A}^{27}$, Vandenberghe $\mathrm{R}^{28,29,30}$, Gerhard $\mathrm{A}^{31,32}$, Butler $\mathrm{CR}^{33}$, Danek $\mathrm{A}^{34}$, Synofzik $\mathrm{M}^{35,36}$, Levin $\mathrm{J}^{34,37}$, Otto $\mathrm{M}^{38}$, Ghidoni $\mathrm{R}^{39}$, Frisoni $\mathrm{G}^{40,41}$, Sorbi $\mathrm{S}^{42,43}$, Georgia Peakman ${ }^{44}$, Emily Todd ${ }^{44}$, Martina Bocchetta ${ }^{44}$, Rohrer $\mathrm{JD}^{44}$, Borroni B ${ }^{1}$, on behalf of the Genetic FTD Initiative (GENFI).\#

*These authors contributed equally to the present work

${ }^{\#}$ List of GENFI Consortium Members in Appendix

${ }^{1}$ Centre for Neurodegenerative Disorders, Department of Clinical and Experimental Sciences, University of Brescia, Brescia, Italy

${ }^{2}$ Stroke Unit, Azienda Socio Sanitaria Territoriale Spedali Civili, Spedali Civili Hospital, Brescia, Italy

${ }^{3}$ Focus Lab, Department of Psychology, University of Turin, Turin, Italy

${ }^{4}$ GCS-FMRI, Koelliker Hospital and Department of Psychology, University of Turin, Turin, Italy

${ }^{5}$ Neuroscience Institute of Turin, University of Turin, Turin, Italy

${ }^{6}$ Neuroradiology Unit, University of Brescia, Italy

${ }^{7}$ Biotechnology Laboratory, Department of Diagnostic, Spedali Civili Hospital, Brescia, Italy

${ }^{8}$ Department of Neurology, Erasmus Medical Center, Rotterdam, Netherlands

${ }^{9}$ Neurology Department, Hospital Clinic, Institut d'Investigacions Biomèdiques, Barcelona, Spain

${ }^{10}$ Department of Neurology, Hospital Universitario Donostia, San Sebastian, Gipuzkoa, Spain

${ }^{11}$ Neuroscience Area, Biodonostia Health Research Institute, San Sebastian, Gipuzkoa, Spain

${ }^{12}$ Neurology Department, Centro Hospitalar e Universitário de Coimbra, Portugal

${ }^{13}$ Faculty of Medicine, University of Coimbra, Coimbra, Portugal

${ }^{14}$ Centre of Neurosciences and Cell biology, Universidade de Coimbra, Coimbra, Portugal

${ }^{15}$ Clinique Interdisciplinaire de Mémoire, Département des Sciences Neurologiques, CHU de Québec, and Faculté de Médecine, Université Laval, QC, Canada

${ }^{16}$ Department of Psychiatry, McGill University Health Centre, McGill University, Montreal, Quebec, Canada

${ }^{17}$ McConnell Brain Imaging Centre, Montreal Neurological Institute, McGill University, Montreal, Québec, Canada

${ }^{18}$ Center for Alzheimer Research, Division of Neurogeriatrics, Department of Neurobiology, Care Sciences and Society, Bioclinicum, Karolinska Institutet, Solna, Sweden

${ }^{19}$ Unit for Hereditary Dementias, Theme Aging, Karolinska University Hospital, Solna, Sweden

${ }^{20}$ Department of Pathophysiology and Transplantation, "Dino Ferrari" Center, University of Milan, Milan, Italy

${ }^{21}$ Fondazione Cà Granda, IRCCS Ospedale Maggiore Policlinico, Milan, Italy

${ }^{22}$ Sunnybrook Health Sciences Centre, Sunnybrook Research Institute, Toronto, ON, Canada

${ }^{23}$ Tanz Centre for Research in Neurodegenerative Diseases, University of Toronto, Toronto, ON, Canada

${ }^{24}$ Department of Clinical Neurosciences, University of Cambridge, Cambridge, UK 
${ }^{25}$ Department of Clinical Neurological Sciences, University of Western Ontario, London, ON, Canada

${ }^{26}$ Fondazione Istituto di Ricovero e Cura a Carattere Scientifico Istituto Neurologico Carlo Besta, Milan, Italy

${ }^{27}$ Laboratory of Neurosciences, Institute of Molecular Medicine, Faculty of Medicine, University of Lisbon, Lisbon, Portugal

${ }^{28}$ Laboratory for Cognitive Neurology, Department of Neurosciences, KU Leuven, Leuven, Belgium

${ }^{29}$ Neurology Service, University Hospitals Leuven, Belgium

${ }^{30}$ Leuven Brain Institute, KU Leuven, Leuven, Belgium

${ }^{31}$ Division of Neuroscience and Experimental Psychology, Wolfson Molecular Imaging Centre, University of Manchester, Manchester, UK

${ }^{32}$ Departments of Geriatric Medicine and Nuclear Medicine, University of Duisburg- Essen, Germany

${ }^{33}$ Nuffield Department of Clinical Neurosciences, Medical Sciences Division, University of Oxford, Oxford, UK

${ }^{34}$ Neurologische Klinik und Poliklinik, Ludwig-Maximilians-Universität, Munich, Germany

${ }^{35}$ Department of Neurodegenerative Diseases, Hertie-Institute for Clinical Brain Research and Center of Neurology, University of Tübingen, Tübingen, Germany

${ }^{36}$ Center for Neurodegenerative Diseases (DZNE), Tübingen, Germany

${ }^{37}$ German Center for Neurodegenerative Diseases (DZNE), Munich, Germany

${ }^{38}$ Department of Neurology, University Hospital Ulm, Ulm, Germany

${ }^{39}$ Molecular Markers Laboratory, IRCCS Istituto Centro San Giovanni di Dio Fatebenefratelli, Brescia, Italy

${ }^{40}$ Istituto di Ricovero e Cura a Carattere Scientifico (IRCCS) Istituto Centro San Giovanni di Dio Fatebenefratelli, Brescia, Italy

${ }^{41}$ Memory Clinic and LANVIE-Laboratory of Neuroimaging of Aging, University Hospitals and University of Geneva, Geneva, Switzerland

${ }^{42}$ Department of Neuroscience, Psychology, Drug Research and Child Health, University of Florence, Florence, Italy

${ }^{43}$ Istituto di Ricovero e Cura a Carattere Scientifico (IRCCS) “Don Gnocchi”, Florence, Italy

${ }^{44}$ Department of Neurodegenerative Disease, Dementia Research Centre, UCL Institute of Neurology, Queen Square, London, UK

Manuscript word count: 2679

\section{Corresponding Author:}

Barbara Borroni, MD

Centre for Neurodegenerative Disorders

Department of Clinical and Experimental Sciences

University of Brescia, Brescia, Italy

Email: bborroni@inwind.it 


\section{Abstract}

Introduction: MRI measures may be used as outcome markers in Frontotemporal dementia (FTD).

Objectives: To predict MRI cortical thickness (CT) at follow-up at the single subject level, using brain MRI acquired at baseline in preclinical FTD.

Methods: 84 presymptomatic subjects carrying Granulin mutations underwent MRI scans at baseline and at follow-up (31.2 \pm 16.5 months). Multivariate nonlinear mixed-effects model was used for estimating individualized CT at followup based on baseline MRI data. The automated user-friendly preGRN-MRI script was coded.

Results: Prediction accuracy was high for each considered brain region (i.e., prefrontal region, real CT at follow-up vs. predicted CT at follow-up, mean error $<=1.87 \%$ ). The sample size required to detect a reduction in decline in a 1 -year clinical trial was equal to 52 subjects (power $=0.80$, alpha $=0.05$ ).

Discussion: The preGRN-MRI tool, using baseline MRI measures, was able to predict the expected MRI atrophy at follow-up in presymptomatic subjects carrying GRN mutations with good performances. This tool could be useful in clinical trials, where deviation of CT from the predicted model may be considered an effect of the intervention itself.

Key-words: Frontotemporal dementia; Granulin; mutation; Magnetic Resonance Imaging; presymptomatic; preclinical. 


\section{Introduction}

Frontotemporal dementia (FTD) refers to a heterogeneous group of disorders predominantly affecting the frontal and temporal lobes and characterised by behavioural disturbances, impairment of executive functions or language deficits $[1,2]$. About $40 \%$ of FTD patients have a family history of dementia, and about $20 \%$ have a clear autosomal dominant inheritance [3]. Among monogenic FTD, mutations in Granulin gene (GRN) are one of the most frequent genetic determinants [4-6] where symptoms are preceded by a long period of gradual accrual of subtle impairment of cognitive functions, progressive brain imaging abnormalities and biomarker changes. However, despite the timely characterisation of early and proximity markers of disease onset $[7,8]$, outcome measures to test the efficacy of treatment interventions are not yet validated. Indeed, in $G R N$ mutation carriers a number of potential therapeutic drugs have been designed $[9,10]$, and it is likely that these drugs may be more effective if administered early in disease course. However, because of the tautologic impossibility to define a clinical outcome for preclinical FTD, our strategic approach is directed towards fluidic or imaging biomarkers as outcome measures.

Circulating progranulin levels have been demonstrated useful to detect the presence of $G R N$ haploinsufficiency [11], while serum or cerebrospinal fluid (CSF) Neurofilaments Light Chain (NfL) or Glial Fibrillary Acidic Protein (GFAP) concentrations may be used to predict early symptoms development $[12,13]$, but their utility as outcome markers need to be further established. In preclinical $G R N$ mutations carriers, cross-sectional studies have identified significant grey matter atrophy and white matter hyperintensities in mutation carriers up to 10 years before expected symptom onset $[7,14,15]$, while longitudinal studies have carefully shown imaging trajectories over time [15-17]. However, none of these markers can be currently used as outcome measures with sufficient accuracy at the single subject level.

Magnetic resonance imaging (MRI) markers are good candidates for the assessment of intervention efficacy, as they may aid to track the delay of disease progression, selectively evaluating effects on specific brain regions. An ideal marker should be able to accurately predict the expected MRI features at follow up, should be reliable and reproducible, and should be consistent across different centres. In other words, a useful outcome MRI marker should be able to forecast a subject's follow-up findings from baseline data. Accordingly, a pharmacological or non-pharmacological intervention may be considered effective when associated with a significant difference from the predicted model, indicating a reduction of expected disease progression. This approach could be used in early experimental medicine studies without placebo/sham treatment, which is particularly useful in view of the neurosurgical intervention required for some proposed $G R N$ treatments and the challenges to global recruitment for large placebo-controlled studies. 
In the present work, we used a multivariate nonlinear mixed effects model to predict brain MRI data at follow-up in presymptomatic subjects carrying pathogenetic $G R N$ mutations from the Genetic FTD Initiative (GENFI) cohort. Such multivariate nonlinear mixed effects models have been used to estimate individualized longitudinal trajectories of neuroimaging data, but few studies examined neurodegenerative disorders [18]. We computed and coded an automated user-friendly script in which the simple and straightforward entry of raw baseline MRI T1-weighted data resulted in an accurate prediction of follow-up MRI data at a given time point and at the single subject level. 


\section{Methods}

Participants. Data for this study were drawn from the GENFI multicentre cohort study (data freeze 5), which consists of 22 research centres across Europe and Canada (www.genfi.org.uk). We included subjects carrying null mutations in the GRN gene at their presymptomatic stage, and for whom both baseline and follow-up MRI was available.

A standardised clinical assessment was performed for each subject, including the Digit Symbol Task, parts A and B of the Trail Making Test, the Wechsler Abbreviated Scale of Intelligence Block Design task, the short version of the Boston Naming Test and Letter and Category fluences [7].

Local ethics committees approved the study at each site and all participants provided written informed consent; the study was conducted according to the Declaration of Helsinki.

Study design. This study was aimed at predicting follow-up brain MRI data at a given time point and at single subject level with the baseline MRI data serving as inputs to the model. We propose that if a treatment intervention is carried out, any deviation from the predicted model may be considered an effect of the intervention itself (see Figure 1).

There were five steps to the study: 1) we extracted cortical thickness maps with a widely used automated brain atlas from baseline and follow-up MRI scans (see below, MRI acquisition and pre-processing section); 2) we run a multivariate nonlinear mixed effects model and an expectation-maximization algorithm for estimating individualized longitudinal trajectories of neuroimaging data (see below, Statistical approach section); 3) we then run a montecarlo simulation (5000 runs) leaving ten random subject out to assess results reliability in predicting follow-up MRI cortical regions from baseline MRI cortical thickness at a single subject level, and we assessed the mean percent error the for each considered brain region; 4) we coded a user friendly toolbox, the preGRN-MRI, to predict MRI at follow-up; 5) we considered baseline MRI data of another cohort of presymptomatic GRN mutations carriers from the GENFI study, not included in the previous analysis, and we assessed the mean percent error in this second dataset to further prove the reliability of the method.

The preGRN-MRI toolbox, the user guide and the related example dataset are available on request (Prof. Barbara Borroni, bborroni@inwind.it).

MRI acquisition and pre-processing. Participants were scanned at their local site on 3 Tesla scanners from two different manufacturers, either Philips Healthcare or Siemens Healthcare Diagnostics. The acquisition protocol, designed to match across scanners as much as possible, included a volumetric T1-weighted MRI scan, as previously published [7]. Baseline and follow-up scans were processed using the standardized longitudinal cortical thickness pipeline of the Computational Anatomy Toolbox (CAT v12.6, http://www.neuro.uni-jena.de/cat/), extension to SPM12 
V.7219 running on MATLAB R2017a). The quality assurance framework implemented in CAT was applied on a scale from $\mathrm{A}$ to $\mathrm{F}$ (A excellent to $\mathrm{F}$ unacceptable/failed), and we only retained scans with an overall quality ranging from $\mathrm{A}$ (excellent) to $\mathrm{C}$ (satisfactory).

Baseline and follow-up cortical thickness maps were parceled into 68 cortical regions, according to the DesikanKilliany atlas [19]. Mean cortical thickness of each region was estimated in the native space. Then, mean values were mediated according to their belonging to brain macro-areas: lateral frontal cortex (caudal middle frontal, pars opercularis, pars orbitalis, pars triangularis, rostral middle frontal), orbitofrontal cortex (lateral orbitofrontal, medial orbitofrontal, frontal pole), superior frontal cortex (superior frontal), insula (insula), lateral temporal cortex (inferior temporal, middle temporal, superior temporal), medial temporal cortex (entorhinal, fusiform, parahippocampal, temporal pole), lateral parietal cortex (inferior parietal, superior parietal) and precuneus (precuneus).

Statistical approach. In this work, we used a multivariate nonlinear mixed effects model developed by Bilgel et al. [18] for estimating the trajectories of biomarkers from longitudinal data in different brain areas. As a hierarchical model, each subject was fitted on its regression, with fitting parameters applied a second-order fitting. At this point, whole-group parameters were used to extrapolate a general trend, considering subjects' age and time spans.

The progression of MRI cortical thickness for each subject $S_{i j}$ was assumed to be as followings:

$$
s_{i j}=\alpha_{i} t_{i j}+\beta_{i}
$$

where $\alpha_{i}$ is the rate of MRI cortical thickness progression, $t_{i j}$ is the age of the subject $i$ at time $j$, and $\beta_{i}$ is the baseline MRI cortical thickness. Then the $K$ biomarker measurements form a vector $y_{i j}$ for subject $i$ at time $j$ is modeled by:

$$
y_{i j}=a s_{i j}+b+\epsilon_{i j}
$$

where $a$ and $b$ are vector and $\epsilon_{i j} N(0, R)$ is the observation noise assumed to be independent and identically distributed across subjects.

This is a mixed effects model that incorporates the fixed effects $a$ and $b$, the individual random effects $\alpha_{i}$ and $\beta_{i}$. The model is nonlinear in the parameters.

To solve for the parameters, we applied an expectation-maximization (EM) algorithm [20], an iterative method that estimates parameters in statistical models, depending on unobserved latent variables. The algorithm alternates between an expectation (E) step, evaluating the expectation of the log-likelihood using the current estimate for the parameters, and a maximization (M) step, which computes the parameters maximizing the function found in the E step. 
To assess how the results of the statistical analysis may be generalizable to an independent data set, and to estimate how accurately the predictive model performs, we used a ten-fold cross-validation. The cross-validation method works by partitioning the data into two subsets: a training set and a testing set. The first set is used to calculate the models, while the second set is used to test the model with a set of data not used to calculate the parameters of the models. To minimize the variability, multiple rounds of cross validation were performed using different sets randomly selected by the original dataset. The validation results were averaged to estimate the performance. In our case, we used a ten-fold cross-validation where in each run we randomly select 10 subjects as testing set and the other subjects were used to estimate the parameters of the models.

The mean error between the predicted cortical thickness of each region and then expected (real) cortical thickness of each region $[100 *($ predicted value - expected value)/predicted value] was calculated.

Finally, we performed a power analysis using $G^{*}$ Power [21], to investigate the minimum number of subjects necessary have a good reliability and power as defined by Cohen [22]. To this, we used the non-centrality parameter $L$ calculated as:

$$
L=f^{2}(n-k-1)
$$

where $n$ is the number of subjects, $k$ the number of regressor and $f^{2}$ defined as:

$$
f^{2}=\frac{R^{2}}{1-R^{2}}
$$

that is the ratio of the proportion of variance accounted for relative to the proportion of variance unaccounted for.

Data availability statement. The data used to support the findings of this study were derived from the Genetic Frontotemporal Dementia Initiative (GENFI, http://genfi. org.uk/). They are available on request from the Principal Investigator of the GENFI consortium (Dr Jonathan Rohrer, University College London, genfi@ucl.ac.uk). 


\section{Results}

Participants. We firstly considered 84 presymptomatic subjects carrying $G R N$ mutations. The mean age was 47.8 (standard deviation, $\mathrm{SD}=11.4), 60.7 \%$ were female $(\mathrm{n}=51)$, with $15.04 \quad(\mathrm{SD}=3.4)$ years of formal schooling. Neuropsychological assessment (mean score \pm standard deviation) was reported in Supplementary Table 1. The mean interval between MRI scans was 31.2 months from the baseline MRI scan ( $\mathrm{SD}=16.5$, range=9.6-71.3 months). MRI quality ranking was excellent/good for both baseline and follow-up scans (baseline: $82 \% \mathrm{~B}, 18 \% \mathrm{~B}$-; follow-up: $80 \% \mathrm{~B}$, $20 \%$ B-).

Baseline and follow-up cortical thickness values are reported in Table 1 (first cohort). We found significant differences between baseline and follow-up in the superior frontal region, bilaterally. These areas presented more severe cortical thinning at follow-up compared to baseline (one-way mixed ANOVA, with baseline vs. follow-up MRI as withinsubjects factors). No other significant differences between baseline and follow-up cortical regions were found.

Model fitting. The prediction analysis takes baseline cortical regions as input, considering age and time between the two MRI scans as regressors, and the model was then fitted. As reported in Figure 2 and Table 1 (first cohort), overall prediction accuracy was high for each considered brain region, with low mean error. The best prediction was obtained considering lateral prefrontal regions, bilaterally (real cortical thickness at follow-up vs. predicted cortical thickness at follow-up, mean error $<=1.87 \%$ ), while the worst was for medial temporal region, bilaterally (mean error $=5.82 \%$ and 6.89\%). Thus, for each subject, we obtained overall high accuracy in predicting brain imaging outcome.

We then coded a user-friendly automated preGRN-MRI toolbox to predict cortical thickness of each region at follow-up at the single subject level. No significant further preprocessing is needed; the user has only to insert the age of the subject at baseline MRI, the values of each considered baseline cortical region, previously computed with the DesikanKilliany atlas 19, and the age of the subject at the desired MRI follow-up. The toolbox will assess automatically the predicted brain regions' values at follow-up in the referral presymptomatic subject carrying a $G R N$ mutation.

Model validation. We further evaluated the effectiveness of our toolbox, using a new dataset of 6 presymptomatic subjects carrying $G R N$ mutations from the GENFI cohort, not included in the previous analyses. The mean age was 47.3 (standard deviation, $\mathrm{SD}=11.4), 33 \%$ were female $(\mathrm{n}=2)$, with $11.4(\mathrm{SD}=4.3)$ years of formal schooling. The mean follow-up MRI was 18.2 months ( $\mathrm{SD}=6.4$, range=12-25 months). MRI quality ranking was excellent/good for both baseline and follow-up scans (baseline: $84 \% \mathrm{~B}, 16 \% \mathrm{~B}$-; follow-up: 85\% B, 15\% B-). This second (validation) cohort presented a different pattern of cortical thickness, as demonstrated by the between-cohort comparisons $\left(1^{\text {st }} v s 2^{\text {nd }}\right.$ cohort 
at baseline and at follow-up), with statistically significant differences between the two cohort at baseline as well as at follow-up (frontal, temporal, parietal and insula regions, bilaterally) (Supplementary Table 2).

We re-run the analyses using the previously coded preGRN-MRI toolbox. The mean percent error was comparable, as reported in Figure 3 and Table 1 (second cohort).

Sample size estimates. The sample size required to detect a reduction in decline of overall cortical thickness in a 1-year clinical trial was equal to 52 subjects (power $=0.80$, alpha $=0.05$ ). 


\section{Discussion}

Our study demonstrated that a novel user-friendly toolbox, preGRN-MRI, can accurately predict cortical thickness changes at follow-up MRI, at the single subject level over a timescale compatible for clinical trials. Thus, preGRN-MRI toolbox might be proposed as a useful device to track neuroimaging changes in early phase clinical trials, as any deviation of cortical thickness from the predicted model could be considered an effect of the intervention itself. It avoids complicated processing by the end users, by providing them with an easy-to-use, automated pipelined toolbox. It also produces comprehensive and interpretable results for model evaluation toward better understanding FTD, and better design of trials.

We considered MRI T1-weighted structural imaging and a widely used atlas [19] to easily compute the data analysis, and to use preGRN-MRI toolbox in any centre with comparable findings, independently of MRI scan or acquisition process $[23,24]$. Indeed, in both the first and in the second cohorts the best prediction was obtained by considering the lateral frontal cortex (mean error $<1.88 \%$ and $<1.20 \%$, respectively) but other regions scored adequately, suggesting the possibility to compute a compound score to increase predictive performances.

These results show that the nonlinear mixed effect model has good reliability and good generalizability. The proposed framework simplifies the estimation of parameters, and accurately estimates the trajectory parameters taking into account inter-individual differences: as hierarchical model, the first level regression of the subjects and the subsequent second level analysis provided a regression over time independent from the single subjects. From this point of view, this can be considered as an experimental "single subject" measure along all times. The high reproducibility was further demonstrated by a second dataset from the GENFI cohort (even including subjects with different cortical thickness pattern), with comparable findings.

Future clinical trials in presymptomatic subjects carrying $G R N$ mutations may benefit from preGRN-MRI toolbox to capture variations in response to a treatment intervention. Up to now, no reliable outcome marker in the preclinical stages of FTD is yet available. The present model fitting may be expanded to other monogenic forms of FTD in their preclinical or symptomatic stages. Indeed, we may argue that the model parameters may differ in C9orf 72 or MAPT mutations, as these are characterized by different disease trajectories and the involvement of selective brain areas [7], in both presymptomatic and symptomatic disease stages.

A number of ongoing pharmacological trials aim to postpone or revert disease onset in monogenic FTD. GRN mutations impair transcription of the gene, leading to levels of progranulin in the serum and CSF that are $>50 \%$ lower than normal from birth $[4,5]$. Therefore, therapeutic approaches may range from either increasing transcription from the normal allele or modulating post-translational mechanisms [25]. Clinical trials have already tested amiodarone, 
with the purpose to increase progranulin levels in GRN mutations carriers, with no clear-cut efficacy [26] and pharmacological trials targeting the sortilin (SORT1), a lysosomal trafficking receptor for progranulin and mediating progranulin endocytosis [27], are ongoing with promising results [28]. Other potential therapeutic strategies are in the pipeline, including the delivery of a healthy $G R N$ gene via an AAV9 vector [29]. Recently, non-pharmacological approaches by using non-invasive brain stimulation technologies have been demonstrated safe and effective in restoring clinical symptoms and brain connectivity in FTD patients [30-32]. Within this context, and prospectively thinking to future intervention strategies, a reliable surrogate marker of disease, such as regional cortical thinning, may be of crucial to assess treatment efficacy in clinical trials. We do not argue against the ultimate necessity for randomized placebo controlled trials. but in view of the low prevalence of known $G R N$ mutation carriers worldwide, and the invasiveness of non-pharmacological approaches in development, early proof of concept studies may benefit from open label studies without placebo, relying on accurate predictive biomarkers to indicate efficacy.

PreGRN-MRI toolbox presents several advantages for this approach. It is automated, together with parameter optimization through nested cross-validation and it is flexible, allowing users to consider different brain regions individually or to compute a comprehensive measure of brain changes.

We acknowledge that this study entails some limitations. First, the prediction model may be improved considering modulators of disease progression, such as cognitive reserve proxy measures $[16,33]$ or computing cerebral regions in a different way. However, the model used here, implemented by an expectation-maximization algorithm, takes into account unobserved latent variables, i.e. variables that do not change over time, such as TMEM106b genotype [33]. Moreover, testing preGRN-MRI toolbox in future clinical trials may prove its reliability in assessing intervention efficacy. Finally, the same approach might be tested in the other pathogenetic mutations, such as MAPT or C9orf72, to develop a user-friendly toolbox tailored on each monogenic FTD-related disorder.

In conclusions, preGRN-MRI tool, using baseline MRI measures, was able to predict the expected MRI at follow-up in presymptomatic subjects carrying GRN mutations with good performances. In future studies, preGRN-MRI tool might be useful in clinical trials, where deviation of $\mathrm{CT}$ from the predicted model may be considered an effect of the intervention itself.

\section{Acknowledgements}

CG and LÖ report grants from Swedish Frontotemporal Dementia Initiative Schörling Foundation; Swedish Research Council: JPND Prefrontals, 2015-02926 ,2018-02754; Swedish Alzheimer foundation; Swedish Brain Foundation; 
Karolinska Institutet Doctoral Funding; KI StratNeuro; Swedish Dementia foundation and Stockholm County Council ALF/Region Stockholm, during the conduct of the study. 


\section{REFERENCES}

[1] Rascovsky K, Hodges JR, Knopman D, Mendez MF, Kramer JH, Neuhaus J, Van Swieten JC, Seelaar H, Dopper EGP, Onyike CU, Hillis AE, Josephs KA, Boeve BF, Kertesz A, Seeley WW, Rankin KP, Johnson JK, Gorno-Tempini ML, Rosen H, Prioleau-Latham CE, Lee A, Kipps CM, Lillo P, Piguet O, Rohrer JD, Rossor MN, Warren JD, Fox NC, Galasko D, Salmon DP, Black SE, Mesulam M, Weintraub S, Dickerson BC, Diehl-Schmid J, Pasquier F, Deramecourt V, Lebert F, Pijnenburg Y, Chow TW, Manes F, Grafman J, Cappa SF, Freedman M, Grossman M, Miller BL (2011) Sensitivity of revised diagnostic criteria for the behavioural variant of frontotemporal dementia. Brain 134, 2456-2477.

[2] Gorno-Tempini ML, Hillis AE, Weintraub S, Kertesz A, Mendez M, Cappa SF, Ogar JM, Rohrer JD, Black S, Boeve BF, Manes F, Dronkers NF, Vandenberghe R, Rascovsky K, Patterson K, Miller BL, Knopman DS, Hodges JR, Mesulam MM, Grossman M (2011) Classification of primary progressive aphasia and its variants. Neurology 76, 1006-1014.

[3] Borroni B, Padovani A (2013) Dementia: A new algorithm for molecular diagnostics in FTLD. Nature Reviews Neurology 9, 241-242.

[4] Cruts M, Gijselinck I, van der Zee J, Engelborghs S, Wils H, Pirici D, Rademakers R, Vandenberghe R, Dermaut B, Martin J-J, van Duijn C, Peeters K, Sciot R, Santens P, De Pooter T, Mattheijssens M, Van den Broeck M, Cuijt I, Vennekens K, De Deyn PP, Kumar-Singh S, Van Broeckhoven C (2006) Null mutations in progranulin cause ubiquitin-positive frontotemporal dementia linked to chromosome 17q21. Nature 442, 920-4.

[5] Baker M, Mackenzie IR, Pickering-Brown SM, Gass J, Rademakers R, Lindholm C, Snowden J, Adamson J, Sadovnick AD, Rollinson S, Cannon A, Dwosh E, Neary D, Melquist S, Richardson A, Dickson D, Berger Z, Eriksen J, Robinson T, Zehr C, Dickey CA, Crook R, McGowan E, Mann D, Boeve B, Feldman H, Hutton M (2006) Mutations in progranulin cause tau-negative frontotemporal dementia linked to chromosome 17. Nature 442, 916-919.

[6] Moore KM, Nicholas J, Grossman M, McMillan CT, Irwin DJ, Massimo L, Van Deerlin VM, Warren JD, Fox NC, Rossor MN, Mead S, Bocchetta M, Boeve BF, Knopman DS, Graff-Radford NR, Forsberg LK, Rademakers R, Wszolek ZK, van Swieten JC, Jiskoot LC, Meeter LH, Dopper EG, Papma JM, Snowden JS, Saxon J, Jones M, Pickering-Brown S, Le Ber I, Camuzat A, Brice A, Caroppo P, Ghidoni R, Pievani M, Benussi L, Binetti G, Dickerson BC, Lucente D, Krivensky S, Graff C, Öijerstedt L, Fallström M, Thonberg H, Ghoshal N, Morris JC, Borroni B, Benussi A, Padovani A, Galimberti D, Scarpini E, Fumagalli GG, Mackenzie IR, Hsiung GYR, Sengdy P, Boxer AL, Rosen H, Taylor JB, Synofzik M, Wilke C, Sulzer P, Hodges JR, Halliday G, Kwok J, Sanchez-Valle R, Lladó A, Borrego-Ecija S, Santana I, Almeida MR, Tábuas-Pereira M, Moreno F, Barandiaran M, Indakoetxea B, Levin J, Danek A, Rowe JB, Cope TE, Otto M, Anderl-Straub S, de Mendonça A, Maruta C, Masellis M, Black SE, Couratier P, Lautrette G, Huey ED, Sorbi S, Nacmias B, Laforce R, Tremblay MPL, Vandenberghe R, Damme P Van, Rogalski EJ, Weintraub S, Gerhard A, Onyike CU, Ducharme S, Papageorgiou SG, Ng ASL, Brodtmann A, Finger E, Guerreiro R, Bras J, Rohrer JD, Heller C, Convery RS, Woollacott IO, Shafei RM, Graff-Radford J, Jones DT, Dheel CM, Savica R, Lapid MI, Baker M, Fields JA, Gavrilova R, Domoto-Reilly K, Poos JM, Van der Ende EL, Panman JL, Donker Kaat L, Seelaar H, Richardson A, Frisoni G, Mega A, Fostinelli S, Chiang HH, Alberici A, Arighi A, Fenoglio C, Heuer H, Miller B, Karydas A, Fong J, João Leitão M, Santiago B, Duro D, Ferreira C, Gabilondo A, De Arriba M, Tainta M, Zulaica M, Ferreira C, Semler E, Ludolph A, Landwehrmeyer B, Volk AE, Miltenberger G, Verdelho A, Afonso S, Tartaglia MC, Freedman M, Rogaeva E, Ferrari C, Piaceri I, Bessi V, Lombardi G, StOnge F, Doré MC, Bruffaerts R, Vandenbulcke M, Van den Stock J, Mesulam MM, Bigio E, Koros C, Papatriantafyllou J, Kroupis C, Stefanis L, Shoesmith C, Robertson E, Coppola G, Da Silva Ramos EM, Geschwind D (2020) Age at symptom onset and death and disease duration in genetic frontotemporal dementia: an international retrospective cohort study. The Lancet Neurology 19, $145-156$. 
[7] Rohrer JD, Nicholas JM, Cash DM, van Swieten J, Dopper E, Jiskoot L, van Minkelen R, Rombouts SA, Cardoso MJ, Clegg S, Espak M, Mead S, Thomas DL, de Vita E, Masellis M, Black SE, Freedman M, Keren R, MacIntosh BJ, Rogaeva E, Tang-Wai D, Tartaglia MC, Laforce R, Tagliavini F, Tiraboschi P, Redaelli V, Prioni S, Grisoli M, Borroni B, Padovani A, Galimberti D, Scarpini E, Arighi A, Fumagalli G, Rowe JB, Coyle-Gilchrist I, Graff C, Fallström M, Jelic V, Ståhlbom AK, Andersson C, Thonberg H, Lilius L, Frisoni GB, Pievani M, Bocchetta M, Benussi L, Ghidoni R, Finger E, Sorbi S, Nacmias B, Lombardi G, Polito C, Warren JD, Ourselin S, Fox NC, Rossor MN (2015) Presymptomatic cognitive and neuroanatomical changes in genetic frontotemporal dementia in the Genetic Frontotemporal dementia Initiative (GENFI) study: A cross-sectional analysis. The Lancet Neurology 14, 253-262.

[8] Benussi A, Gazzina S, Premi E, Cosseddu M, Archetti S, Dell'Era V, Cantoni V, Cotelli MS, Alberici A, Micheli A, Benussi L, Ghidoni R, Padovani A, Borroni B (2019) Clinical and biomarker changes in presymptomatic genetic frontotemporal dementia. Neurobiology of Aging 76, 133-140.

[9] Greaves C V., Rohrer JD (2019) An update on genetic frontotemporal dementia. Journal of Neurology 266, 2075-2086.

[10] Galimberti D, Fenoglio C, Scarpini E (2018) Progranulin as a therapeutic target for dementia. Expert Opinion on Therapeutic Targets 22, 579-585.

[11] Ghidoni R, Benussi L, Glionna M, Neurology MF-, 2008 U (2008) Low plasma progranulin levels predict progranulin mutations in frontotemporal lobar degeneration. AAN Enterprises 71, 12351239.

[12] van der Ende EL, Meeter LH, Poos JM, Panman JL, Jiskoot LC, Dopper EGP, Papma JM, de Jong FJ, Verberk IMW, Teunissen C, Rizopoulos D, Heller C, Convery RS, Moore KM, Bocchetta M, Neason M, Cash DM, Borroni B, Galimberti D, Sanchez-Valle R, Laforce R, Moreno F, Synofzik M, Graff C, Masellis M, Carmela Tartaglia M, Rowe JB, Vandenberghe R, Finger E, Tagliavini F, de Mendonça A, Santana I, Butler C, Ducharme S, Gerhard A, Danek A, Levin J, Otto M, Frisoni GB, Cappa S, Pijnenburg YAL, Rohrer JD, van Swieten JC (2019) Serum neurofilament light chain in genetic frontotemporal dementia: a longitudinal, multicentre cohort study. The Lancet Neurology 18, 1103-1111.

[13] Heller C, Foiani MS, Moore K, Convery R, Bocchetta M, Neason M, Cash DM, Thomas D, Greaves CV, Woollacott IOC, Shafei R, van Swieten JC, Moreno F, Sanchez-Valle R, Borroni B, Laforce R, Masellis M, Tartaglia MC, Graff C, Galimberti D, Rowe JB, Finger E, Synofzik M, Vandenberghe R, de Mendonca A, Tagliavini F, Santana I, Ducharme S, Butler CR, Gerhard A, Levin J, Danek A, Frisoni G, Sorbi S, Otto M, Heslegrave AJ, Zetterberg H, Rohrer JD, Rossor MN, Warren JD, Fox NC, Guerreiro R, Bras J, Nicholas J, Mead S, Jiskoot L, Meeter L, Panman J, Papma J, van Minkelen R, Pijnenburg Y, Barandiaran M, Indakoetxea B, Gabilondo A, Tainta M, de Arriba M, Gorostidi A, Zulaica M, Villanua J, Diaz Z, Borrego-Ecija S, Olives J, Lladó A, Balasa M, Antonell A, Bargallo N, Premi E, Cosseddu M, Gazzina S, Padovani A, Gasparotti R, Archetti S, Black S, Mitchell S, Rogaeva E, Freedman M, Keren R, Tang-Wai D, Öijerstedt L, Andersson C, Jelic V, Thonberg H, Arighi A, Fenoglio C, Scarpini E, Fumagalli G, Cope T, Timberlake C, Rittman T, Shoesmith C, Bartha R, Rademakers R, Wilke C, Karnarth H-O, Bender B, Bruffaerts R, Vandamme P, Vandenbulcke M, Ferreira CB, Miltenberger G, Maruta C, Verdelho A, Afonso S, Taipa R, Caroppo P, di Fede G, Giaccone G, Prioni S, Redaelli V, Rossi G, Tiraboschi P, Dura D, Almeida MR, Castelo-Branco M, João Leitão M, Tabuas-Pereira M, Santiago B, Gauthier S, Rosa-Neto P, Veldsman M, Flanagan T, Prix C, Hoegen T, Wlasich E, Loosli S, Schonecker S, Semler E, Anderl-Straub S, Benussi L, Binetti G, Ghidoni R, Pievani M, Lombardi G, Nacmias B, Ferrari C, Bessi V (2020) Plasma glial fibrillary acidic protein is raised in progranulin-associated frontotemporal dementia. Journal of Neurology, Neurosurgery and Psychiatry 91,. 
[14] Paternicò D, Premi E, Gazzina S, Cosseddu M, Alberici A, Archetti S, Cotelli MS, Micheli A, Turla M, Gasparotti R, Padovani A, Borroni B (2016) White matter hyperintensities characterize monogenic frontotemporal dementia with granulin mutations. Neurobiology of aging 38, 176-180.

[15] Sudre CH, Bocchetta M, Heller C, Convery R, Neason M, Moore KM, Cash DM, Thomas DL, Woollacott IOC, Foiani M, Heslegrave A, Shafei R, Greaves C, van Swieten J, Moreno F, SanchezValle R, Borroni B, Laforce R, Masellis M, Tartaglia MC, Graff C, Galimberti D, Rowe JB, Finger E, Synofzik M, Vandenberghe R, de Mendonça A, Tagliavini F, Santana I, Ducharme S, Butler C, Gerhard A, Levin J, Danek A, Frisoni GB, Sorbi S, Otto M, Zetterberg H, Ourselin S, Cardoso MJ, Rohrer JD (2019) White matter hyperintensities in progranulin-associated frontotemporal dementia: A longitudinal GENFI study. NeuroImage: Clinical 24,.

[16] Gazzina S, Grassi M, Premi E, Cosseddu M, Alberici A, Archetti S, Gasparotti R, Van Swieten J, Galimberti D, Sanchez-Valle R, Laforce RJ, Moreno F, Synofzik M, Graff C, Masellis M, Tartaglia MC, Rowe JB, Vandenberghe R, Finger E, Tagliavini F, de Mendonça A, Santana I, Butler CR, Ducharme S, Gerhard A, Danek A, Levin J, Otto M, Frisoni G, Sorbi S, Padovani A, Rohrer JD, Borroni B, Genetic FTD Initiative, GENFI (2019) Education modulates brain maintenance in presymptomatic frontotemporal dementia. Journal of neurology, neurosurgery, and psychiatry $\mathbf{9 0}$, 1124-1130.

[17] Chen Q, Boeve BF, Senjem M, Tosakulwong N, Lesnick T, Brushaber D, Dheel C, Fields J, Forsberg L, Gavrilova R, Gearhart D, Graff-Radford J, Graff-Radford N, Jack CR, Jones D, Knopman D, Kremers WK, Lapid M, Rademakers R, Ramos EM, Syrjanen J, Boxer AL, Rosen H, Wszolek ZK, Kantarci K (2020) Trajectory of lobar atrophy in asymptomatic and symptomatic GRN mutation carriers: a longitudinal MRI study. Neurobiology of Aging 88, 42-50.

[18] Bilgel M, Prince JL, Wong DF, Resnick SM, Jedynak BM (2016) A multivariate nonlinear mixed effects model for longitudinal image analysis: Application to amyloid imaging. NeuroImage 134, 658-670.

[19] Desikan RS, Ségonne F, Fischl B, Quinn BT, Dickerson BC, Blacker D, Buckner RL, Dale AM, Maguire RP, Hyman BT, Albert MS, Killiany RJ (2006) An automated labeling system for subdividing the human cerebral cortex on MRI scans into gyral based regions of interest. NeuroImage 31, 968-980.

[20] Dempster A, Laird N, Rubin D (1977) Maximum Likelihood from Incomplete Data Via the EM Algorithm. Journal of the Royal Statistical Society Series B (Methodological) 39, 1-38.

[21] Faul F, Erdfelder E, Lang AG, Buchner A (2007) G*Power 3: A flexible statistical power analysis program for the social, behavioral, and biomedical sciences. In Behavior Research Methods Psychonomic Society Inc., pp. 175-191.

[22] Cohen J (2013) Statistical Power Analysis for the Behavioral Sciences.

[23] Pievani M, Paternicò D, Benussi L, Binetti G, Orlandini A, Cobelli M, Magnaldi S, Ghidoni R, Frisoni GB (2014) Pattern of structural and functional brain abnormalities in asymptomatic granulin mutation carriers. Alzheimer's \& dementia : the journal of the Alzheimer's Association 10, S354S363.e1.

[24] Gazzina S, Benussi A, Premi E, Paternicò D, Cristillo V, Dell'Era V, Cosseddu M, Archetti S, Alberici A, Gasparotti R, Padovani A, Borroni B (2018) Neuroanatomical Correlates of Transcranial Magnetic Stimulation in Presymptomatic Granulin Mutation Carriers. Brain topography 31, 488-497.

[25] Panza F, Lozupone M, Seripa D, Daniele A, Watling M, Giannelli G, Imbimbo BP (2020) Development of disease-modifying drugs for frontotemporal dementia spectrum disorders. Nature Reviews Neurology 16, 213-228.

[26] Alberici A, Archetti S, Pilotto A, Premi E, Cosseddu M, Bianchetti A, Semeraro F, Salvetti M, Muiesan ML, Padovani A, Borroni B (2014) Results from a pilot study on amiodarone administration in monogenic frontotemporal dementia with granulin mutation. Neurological sciences : official journal of the Italian Neurological Society and of the Italian Society of Clinical Neurophysiology 35, 1215-9. 
[27] Lee WC, Almeida S, Prudencio M, Caulfield TR, Zhang YJ, Tay WM, Bauer PO, Chew J, Sasaguri H, Jansen-west KR, Gendron TF, Stetler CT, Finch NC, Mackenzie IR, Rademakers R, Gao FB, Petrucelli L (2014) Targeted manipulation of the sortilin-progranulin axis rescues progranulin haploinsufficiency. Human Molecular Genetics 23, 1467-1478.

[28] Alector Announces Data from On-going Phase 1b Trial Demonstrating that AL001 Reverses Progranulin Deficiency in Frontotemporal Dementia Patients | BioSpace, Last updated 2019, Accessed on 2019.

[29] Prevail Therapeutics Announces FDA Orphan Drug Designation Granted to PR006 for the Treatment of the Patients with Frontotemporal Dementia with a GRN Mutation, Last updated 2019, Accessed on 2019.

[30] Tsapkini K, Webster KT, Ficek BN, Desmond JE, Onyike CU, Rapp B, Frangakis CE, Hillis AE (2018) Electrical brain stimulation in different variants of primary progressive aphasia: A randomized clinical trial. Alzheimer's \& dementia (New York, $N$ Y) 4, 461-472.

[31] Ficek BN, Wang Z, Zhao Y, Webster KT, Desmond JE, Hillis AE, Frangakis C, Vasconcellos Faria A, Caffo B, Tsapkini K (2018) The effect of tDCS on functional connectivity in primary progressive aphasia. NeuroImage Clinical 19, 703-715.

[32] Benussi A, Dell'era V, Cosseddu M, Cantoni V, Cotelli MS, Cotelli M, Manenti R, Benussi L, Brattini C, Alberici A, Borroni B (2020) Transcranial stimulation in frontotemporal dementia: A randomized, double-blind, sham-controlled trial. Alzheimer's and Dementia: Translational Research and Clinical Interventions 6, 1-11.

[33] Premi E, Grassi M, van Swieten J, Galimberti D, Graff C, Masellis M, Tartaglia C, Tagliavini F, Rowe JB, Laforce R, Finger E, Frisoni GB, de Mendonça A, Sorbi S, Gazzina S, Cosseddu M, Archetti S, Gasparotti R, Manes M, Alberici A, Cardoso MJ, Bocchetta M, Cash DM, Ourselin S, Padovani A, Rohrer JD, Borroni B (2017) Cognitive reserve and TMEM106B genotype modulate brain damage in presymptomatic frontotemporal dementia: A GENFI study. Brain 140, 1784-1791. 
Table 1. Cortical thickness of each brain region in presymptomatic GRN mutation carriers.

\begin{tabular}{|c|c|c|c|c|c|c|c|c|}
\hline \multirow{2}{*}{ Brain ROIs } & \multicolumn{4}{|c|}{ First cohort $(n=84)$} & \multicolumn{4}{|c|}{ Second cohort $(n=6)$} \\
\hline & baseline MRI & $\begin{array}{l}\text { follow-up } \\
\text { MRI }\end{array}$ & p-value & $\begin{array}{c}\text { mean error } \\
\%^{*}\end{array}$ & baseline MRI & $\begin{array}{l}\text { follow-up } \\
\text { MRI }\end{array}$ & p-value & $\begin{array}{c}\text { mean error } \\
\% *\end{array}$ \\
\hline lateral frontal $L$ & $2.76 \pm 0.13$ & $2.76 \pm 0.12$ & 0.71 & $1.84 \pm 0.36$ & $2.88 \pm 0.09$ & $2.85 \pm 0.11$ & 0.52 & $1.19 \pm 0.35$ \\
\hline lateral frontal $R$ & $2.76 \pm 0.12$ & $2.76 \pm 0.17$ & 0.36 & $1.87 \pm 0.49$ & $2.85 \pm 0.12$ & $2.84 \pm 0.10$ & 0.87 & $0.53 \pm 0.13$ \\
\hline orbitofrontal L & $2.65 \pm 0.17$ & $2.67 \pm 0.16$ & 0.84 & $4.07 \pm 0.94$ & $2.82 \pm 0.18$ & $2.77 \pm 0.16$ & 0.63 & $1.84 \pm 0.26$ \\
\hline orbitofrontal $\mathbf{R}$ & $2.64 \pm 0.17$ & $2.67 \pm 0.20$ & 0.06 & $3.69 \pm 0.88$ & $2.86 \pm 0.124$ & $2.86 \pm 0.17$ & 1.00 & $1.95 \pm 0.37$ \\
\hline superior frontal L & $2.88 \pm 0.14$ & $2.83 \pm 0.18$ & $<0.001$ & $2.59 \pm 0.53$ & $3.04 \pm 0.09$ & $3.03 \pm 0.11$ & 0.87 & $1.37 \pm 0.27$ \\
\hline superior frontal $\mathbf{R}$ & $2.87 \pm 0.14$ & $2.82 \pm 0.17$ & $<0.001$ & $2.84 \pm 0.59$ & $3.04 \pm 0.11$ & $3.02 \pm 0.11$ & 0.63 & $0.96 \pm 0.19$ \\
\hline medial temporal $L$ & $3.16 \pm 0.26$ & $3.20 \pm 0.25$ & 0.10 & $5.92 \pm 1.18$ & $3.51 \pm 0.23$ & $3.61 \pm 0.20$ & 0.42 & $4.61 \pm 0.87$ \\
\hline medial temporal $\mathbf{R}$ & $3.18 \pm 0.29$ & $3.22 \pm 0.3$ & 0.08 & $6.89 \pm 1.37$ & $3.61 \pm 0.08$ & $3.54 \pm 0.08$ & 0.20 & $2.11 \pm 0.49$ \\
\hline lateral temporal $\mathrm{L}$ & $2.90 \pm 0.16$ & $2.93 \pm 0.14$ & 0.014 & $2.68 \pm 0.49$ & $2.98 \pm 0.10$ & $2.94 \pm 0.11$ & 0.42 & $1.29 \pm 0.33$ \\
\hline lateral temporal $\mathbf{R}$ & $2.93 \pm 0.15$ & $2.97 \pm 0.20$ & 0.008 & $2.54 \pm 0.62$ & $2.99 \pm 0.14$ & $2.98 \pm 0.15$ & 0.75 & $0.87 \pm 0.14$ \\
\hline lateral parietal $L$ & $2.46 \pm 0.12$ & $2.46 \pm 0.10$ & 0.86 & $2.23 \pm 0.51$ & $2.53 \pm 0.07$ & $2.53 \pm 0.10$ & 0.75 & $1.11 \pm 0.15$ \\
\hline lateral parietal $\mathbf{R}$ & $2.45 \pm 0.14$ & $2.47 \pm 0.16$ & 0.16 & $2.66 \pm 0.61$ & $2.52 \pm 0.07$ & $2.50 \pm 0.10$ & 0.87 & $1.21 \pm 0.19$ \\
\hline precuneus & $2.48 \pm 0.13$ & $2.45 \pm 0.14$ & 0.02 & $2.41 \pm 0.56$ & $2.60 \pm 0.09$ & $2.61 \pm 0.08$ & 0.87 & $1.51 \pm 0.27$ \\
\hline insula $\mathbf{L}$ & $3.49 \pm 0.22$ & $3.51 \pm 0.20$ & 0.14 & $4.44 \pm 0.82$ & $3.69 \pm 0.11$ & $3.63 \pm 0.16$ & 0.42 & $2.40 \pm 0.69$ \\
\hline insula $\mathbf{R}$ & $3.47 \pm 0.22$ & $3.5 \pm 0.26$ & 0.04 & $4.75 \pm 1.06$ & $3.70 \pm 0.25$ & $3.76 \pm 0.18$ & 0.42 & $2.84 \pm 0.61$ \\
\hline
\end{tabular}

$\mathrm{L}=$ left; $\mathrm{R}=$ right; ROIs $=$ Region of Interest expressed in millimeters; significant $\mathrm{p}$-values in bold face, baseline MRI scores vs. follow-up MRI cortical thickness scores ( $\mathrm{p}<0.004$ corrected for multiple comparisons). Results are expressed as mean \pm standard deviation.

* mean error between real cortical thickness at follow-up and predicted cortical thickness at follow-up 


\section{Legend to Figures}

\section{Figure 1. Study design.}

The cartoon depicts the rationale of the present study.

Predicting follow-up MRI data by baseline MRI data input at single subject level may represent an helpful outcome marker of treatment response (first row). To this, we applied multivariate nonlinear mixed effects model and ten-fold cross-validation to predict follow-up MRI at single subject level at a given time point (second row). When treatment intervention is administered, efficacy may be proven by any deviation from the expected MRI scan (third row).

Figure 2. Mean percent error of multivariate nonlinear mixed effects model in predicting follow-up cortical regions by baseline MRI data in first cohort of presymptomatic Granulin (GRN) mutation carriers.

$\mathrm{L}=$ left; $\mathrm{R}=$ right. The results are expressed as mean error \pm standard deviation.

Figure 3. Percent error of multivariate nonlinear mixed effects model in predicting follow-up cortical regions by baseline MRI data in a new sample of 6 presymptomatic Granulin (GRN) mutation carriers.

$\mathrm{L}=$ left; $\mathrm{R}=$ right. High percentage error values for several subjects (subject 6 for left insula, subject 4 for right insula, subject 1 for left medial temporal region) were reported to maintain consistency in the graphical representation. 
Figures

Figure 1.

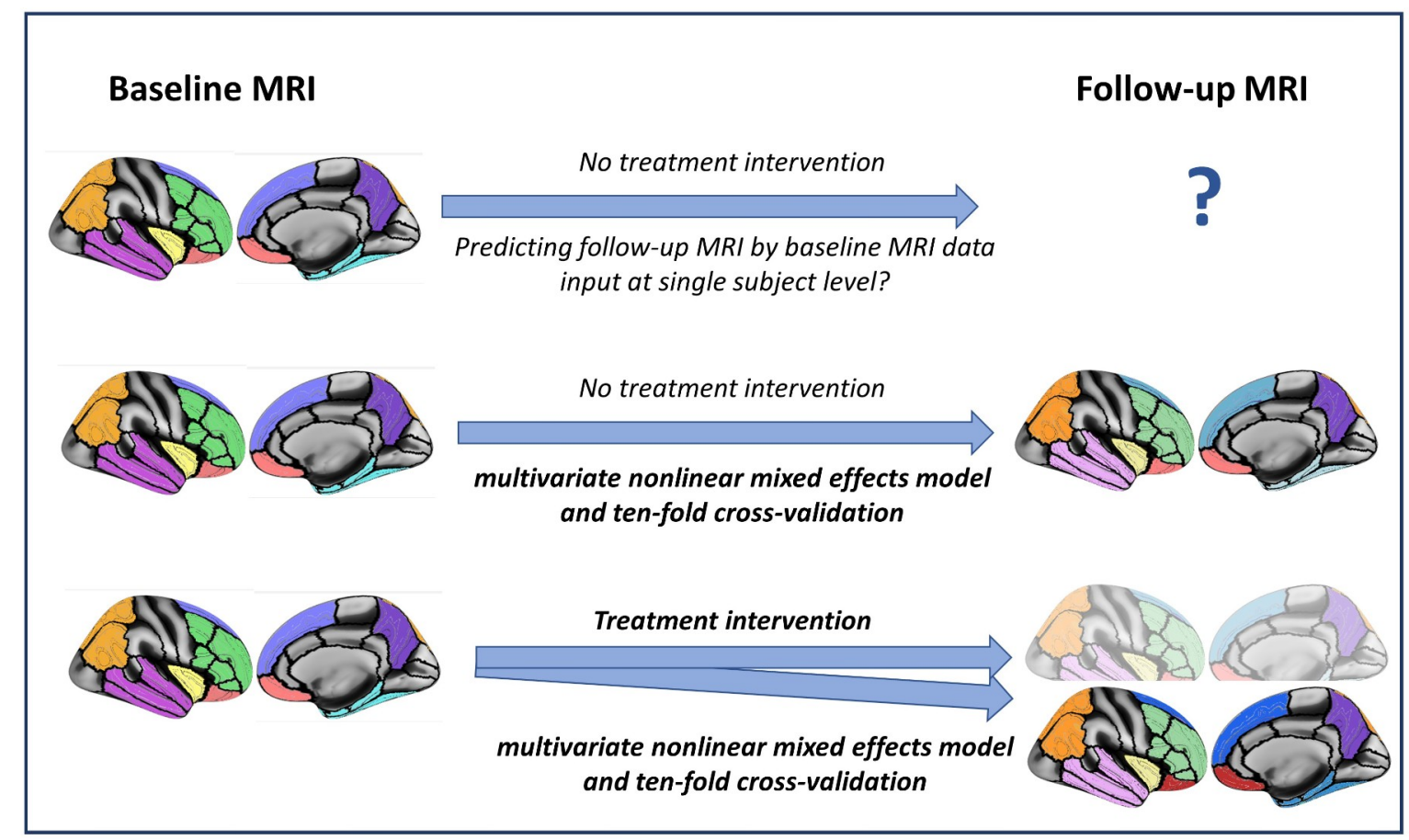


Figure 2.

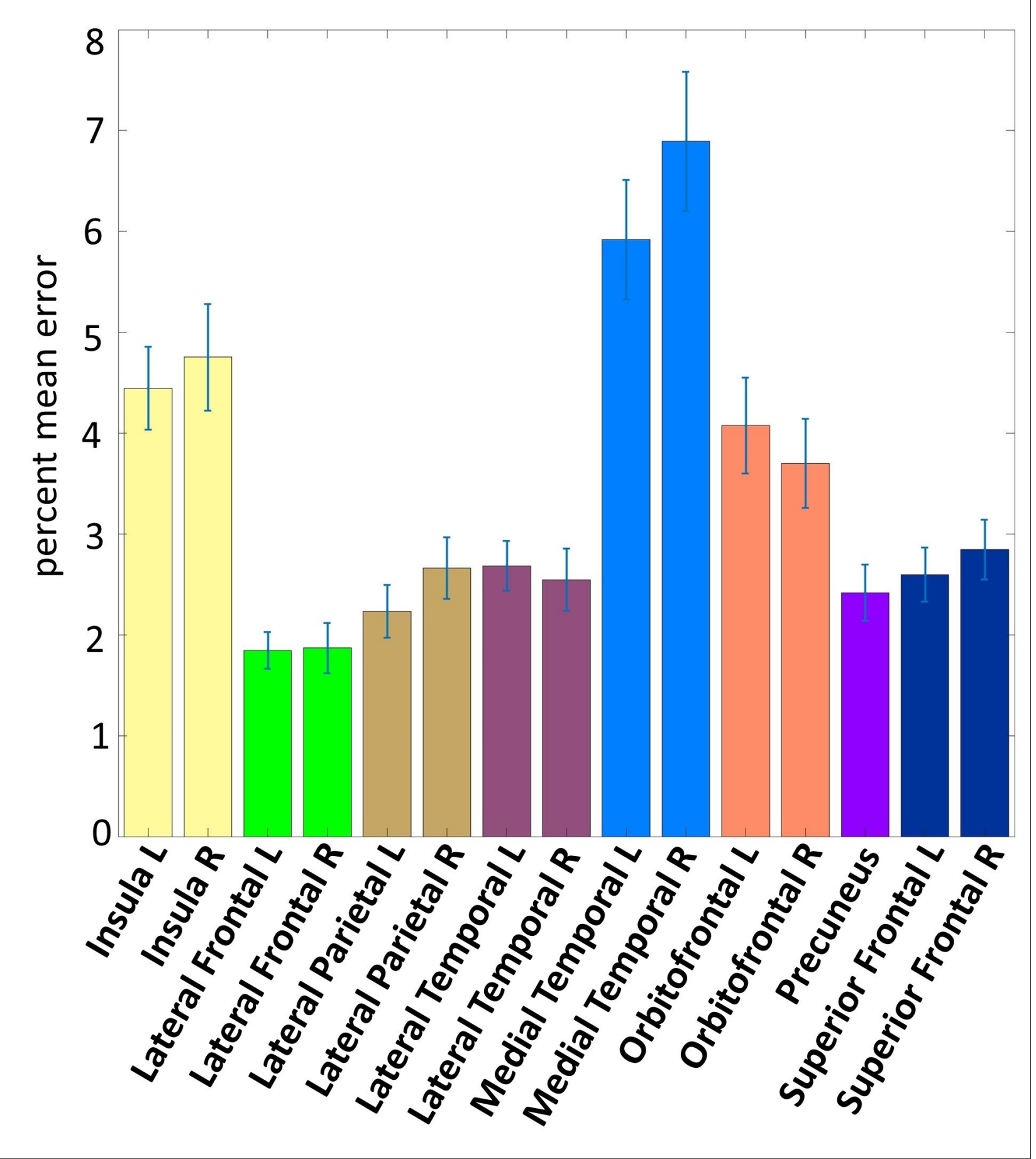


Figure 3.

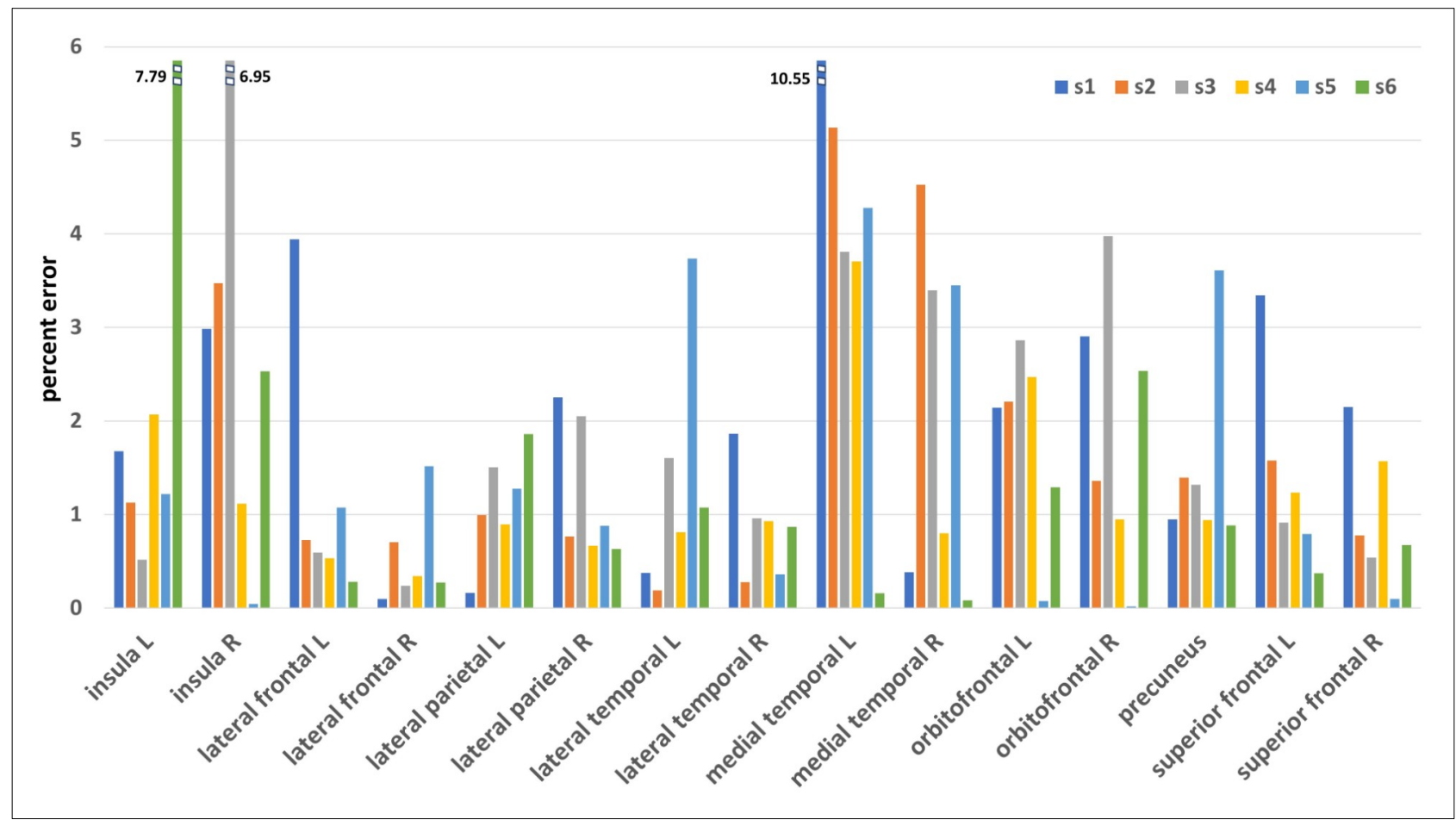


Supplementary Table 1. Standardised neuropsychological assessment in presymptomatic GRN mutation carriers.

\begin{tabular}{lc}
\hline & presymptomatic $\boldsymbol{G R N}$ mutation carriers * \\
\hline Digit Symbol Task & $55.5 \pm 11.8$ \\
Trail Making Test (part A) & $28.7 \pm 10.5$ \\
Trail Making Test (part B) & $64.2 \pm 24.8$ \\
Block Design & $46.1 \pm 15.0$ \\
Boston Naming Test (short) & $28.4 \pm 1.6$ \\
Letter Fluency (combined) & $45.6 \pm 15.1$ \\
Category Fluency (animals) & $25.1 \pm 5.2$ \\
\hline
\end{tabular}

GRN: Granulin mutation carriers; * mean value \pm standard deviation.

Supplementary Table 2. Between-cohort analyses (first cohort vs second cohort).

\begin{tabular}{lcc}
\hline Brain ROIs & Baseline $\left(1^{\text {st }} \mathrm{vs} 2^{\text {nd }}\right.$ cohort $)$ & Follow-up $\left(1^{\text {st }} \mathrm{vs} 2^{\text {nd }}\right.$ cohort $)$ \\
\hline lateral_frontal_L & $0.04^{* * * *}$ & 0.17 \\
lateral_frontal_R & 0.13 & 0.19 \\
orbitofrontal_L & $0.08^{*}$ & 0.22 \\
orbitofrontal_R & $0.02^{* * * * * *}$ & $0.04^{* * * *}$ \\
superior_frontal_L & $0.03^{* * * *}$ & $0.02^{* * * *}$ \\
superior_frontal_R & $0.02^{* * * *}$ & $0.02^{* * * *}$ \\
medial_temporal_L & $0.02^{* * * *}$ & $0.02^{* * * *}$ \\
medial_temporal_R & $0.01^{* * * *}$ & $0.02^{* * * *}$ \\
lateral_temporal_L & 0.19 & 0.9 \\
lateral_temporal_R & 0.44 & 0.9 \\
lateral_parietal_L & 0.22 & 0.22 \\
lateral_parietal_R & 0.19 & 0.48 \\
precuneus & $0.05^{* * * *}$ & $0.02^{* * * *}$ \\
insula_L & $0.04^{* * * *}$ & 0.22 \\
insula_R & $0.08^{*}$ & $0.02^{* * * *}$ \\
\hline
\end{tabular}

ROIs: Rogions of Interests; L: left; R: right;

$* \mathrm{p}<0.05$ uncorrected for multiple comparisons; $* * \mathrm{p}<0.05$ FDR-corrected for multiple comparisons (Mann-Whitney test). 


\section{Appendix. GENFI Consortium Members}

- Sónia Afonso - Instituto Ciencias Nucleares Aplicadas a Saude, Universidade de Coimbra, Coimbra, Portugal;

- Maria Rosario Almeida - Faculty of Medicine, University of Coimbra, Coimbra, Portugal;

- Sarah Anderl-Straub - Department of Neurology, University of Ulm, Ulm, Germany;

- Christin Andersson - Department of Clinical Neuroscience, Karolinska Institutet, Stockholm, Sweden;

- Anna Antonell - Alzheimer's disease and Other Cognitive Disorders Unit, Neurology Service, Hospital Clínic, Barcelona, Spain;

- $\quad$ Andrea Arighi - Fondazione IRCCS Ca' Granda Ospedale Maggiore Policlinico, Neurodegenerative Diseases Unit, Milan, Italy; University of Milan, Centro Dino Ferrari, Milan, Italy;

- Mircea Balasa - Alzheimer's disease and Other Cognitive Disorders Unit, Neurology Service, Hospital Clínic, Barcelona, Spain;

- Myriam Barandiaran - Cognitive Disorders Unit, Department of Neurology, Donostia University Hospital, San Sebastian, Gipuzkoa, Spain; Neuroscience Area, Biodonostia Health Research Insitute, San Sebastian, Gipuzkoa, Spain;

- $\quad$ Nuria Bargalló - Imaging Diagnostic Center, Hospital Clínic, Barcelona, Spain;

- Robart Bartha - Department of Medical Biophysics, The University of Western Ontario, London, Ontario, Canada; Centre for Functional and Metabolic Mapping, Robarts Research Institute, The University of Western Ontario, London, Ontario, Canada;

- Benjamin Bender -Department of Diagnostic and Interventional Neuroradiology, University of Tübingen, Tübingen, Germany;

- Luisa Benussi - Istituto di Ricovero e Cura a Carattere Scientifico Istituto Centro San Giovanni di Dio Fatebenefratelli, Brescia, Italy;

- Valentina Bessi - Department of Neuroscience, Psychology, Drug Research, and Child Health, University of Florence, Florence, Italy;

- Giuliano Binetti - Istituto di Ricovero e Cura a Carattere Scientifico Istituto Centro San Giovanni di Dio Fatebenefratelli, Brescia, Italy;

- Sandra Black -Sunnybrook Health Sciences Centre, Sunnybrook Research Institute, University of Toronto, Toronto, Canada;

- Sergi Borrego-Ecija - Alzheimer's disease and Other Cognitive Disorders Unit, Neurology Service, Hospital Clínic, Barcelona, Spain;

- Jose Bras - Dementia Research Institute, Department of Neurodegenerative Disease, UCL Institute of Neurology, Queen Square, London, UK;

- Rose Bruffaerts - Laboratory for Cognitive Neurology, Department of Neurosciences, KU Leuven, Leuven, Belgium;

- Paola Caroppo - Fondazione IRCCS Istituto Neurologico Carlo Besta, Milano, Italy;

- David Cash - Dementia Research Centre, Department of Neurodegenerative Disease, UCL Institute of Neurology, Queen Square, London, UK;

- Miguel Castelo-Branco - Faculty of Medicine, University of Coimbra, Coimbra, Portugal;

- Rhian Convery - Dementia Research Centre, Department of Neurodegenerative Disease, UCL Institute of Neurology, Queen Square, London, UK;

- $\quad$ Thomas Cope - Department of Clinical Neuroscience, University of Cambridge, Cambridge, UK;

- María de Arriba - Neuroscience Area, Biodonostia Health Research Insitute, San Sebastian, Gipuzkoa, Spain;

- Giuseppe Di Fede -Fondazione IRCCS Istituto Neurologico Carlo Besta, Milano, Italy;

- Zigor Díaz - CITA Alzheimer, San Sebastian, Gipuzkoa, Spain;

- Diana Duro - Faculty of Medicine, University of Coimbra, Coimbra, Portugal;

- Chiara Fenoglio - Fondazione IRCCS Ca' Granda Ospedale Maggiore Policlinico, Neurodegenerative Diseases Unit, Milan, Italy; University of Milan, Centro Dino Ferrari, Milan, Italy;

- Camilla Ferrari - Department of Neuroscience, Psychology, Drug Research, and Child Health, University of Florence, Florence, Italy;

- Catarina B. Ferreira -Laboratory of Neurosciences, Institute of Molecular Medicine, Faculty of Medicine, University of Lisbon, Lisbon, Portugal;

- Nick Fox - Dementia Research Centre, Department of Neurodegenerative Disease, UCL Institute of Neurology, Queen Square, London, UK;

- Morris Freedman -Baycrest Health Sciences, Rotman Research Institute, University of Toronto, Toronto, Canada;

- Giorgio Fumagalli -Fondazione IRCCS Ca' Granda Ospedale Maggiore Policlinico, Neurodegenerative Diseases Unit, Milan, Italy; University of Milan, Centro Dino Ferrari, Milan, Italy; Department of 
Neurosciences, Psychology, Drug Research and Child Health (NEUROFARBA), University of Florence, Florence, Italy;

- Alazne Gabilondo -Neuroscience Area, Biodonostia Health Research Insitute, San Sebastian, Gipuzkoa, Spain;

- Serge Gauthier - Alzheimer Disease Research Unit, McGill Centre for Studies in Aging, Department of Neurology \& Neurosurgery, McGill University, Montreal, Québec, Canada;

- Giorgio Giaccone - Fondazione IRCCS Istituto Neurologico Carlo Besta, Milano, Italy;

- Ana Gorostidi - Neuroscience Area, Biodonostia Health Research Insitute, San Sebastian, Gipuzkoa, Spain;

- Caroline Greaves - Dementia Research Centre, Department of Neurodegenerative Disease, UCL Institute of Neurology, Queen Square, London, UK;

- Rita Guerreiro - Dementia Research Institute, Department of Neurodegenerative Disease, UCL Institute of Neurology, Queen Square, London, UK;

- Carolin Heller - Dementia Research Centre, Department of Neurodegenerative Disease, UCL Institute of Neurology, Queen Square, London, UK;

- Tobias Hoegen -Neurologische Klinik, Ludwig-Maximilians-Universität München, Munich, Germany;

- Begoña Indakoetxea - Cognitive Disorders Unit, Department of Neurology, Donostia University Hospital, San Sebastian, Gipuzkoa, Spain; Neuroscience Area, Biodonostia Health Research Insitute, San Sebastian, Gipuzkoa, Spain;

- Vesna Jelic - Division of Clinical Geriatrics, Karolinska Institutet, Stockholm, Sweden;

- $\quad$ Lize Jiskoot - Department of Neurology, Erasmus Medical Center, Rotterdam, Netherlands;

- Hans-Otto Karnath - Division of Neuropsychology, Hertie-Institute for Clinical Brain Research and Center of Neurology, University of Tübingen, Tübingen, Germany;

- $\quad$ Ron Keren -The University Health Network, Toronto Rehabilitation Institute, Toronto, Canada;

- Tobias Langheinrich - Division of Neuroscience and Experimental Psychology Wolfson Molecular Imaging Centre, University of Manchester, Manchester, UK;

- Maria João Leitão - Centre of Neurosciences and Cell Biology, Universidade de Coimbra, Coimbra, Portugal;

- Albert Lladó - Alzheimer's disease and Other Cognitive Disorders Unit, Neurology Service, Hospital Clínic, Barcelona, Spain;

- Gemma Lombardi - Department of Neuroscience, Psychology, Drug Research and Child Health, University of Florence, Florence, Italy;

- Sandra Loosli -Neurologische Klinik, Ludwig-Maximilians-Universität München, Munich, Germany;

- Carolina Maruta -Laboratory of Language Research, Centro de Estudos Egas Moniz, Faculty of Medicine, University of Lisbon, Lisbon, Portugal;

- Simon Mead - MRC Prion Unit, Department of Neurodegenerative Disease, UCL Institute of Neurology, Queen Square, London, UK;

- $\quad$ Lieke Meeter - Department of Neurology, Erasmus Medical Center, Rotterdam, Netherlands;

- Gabriel Miltenberger - Faculty of Medicine, University of Lisbon, Lisbon, Portugal;

- Rick van Minkelen - Department of Clinical Genetics, Erasmus Medical Center, Rotterdam, Netherlands;

- Sara Mitchell - Sunnybrook Health Sciences Centre, Sunnybrook Research Institute, University of Toronto, Toronto, Canada;

- $\quad$ Katrina Moore - Dementia Research Centre, Department of Neurodegenerative Disease, UCL Institute of Neurology, Queen Square, London UK;

- Benedetta Nacmias - Department of Neuroscience, Psychology, Drug Research and Child Health, University of Florence, Florence, Italy;

- Jennifer Nicholas - Department of Medical Statistics, London School of Hygiene and Tropical Medicine, London, UK;

- Linn Öijerstedt - Department of Geriatric Medicine, Karolinska University Hospital-Huddinge, Stockholm, Sweden;

- Jaume Olives - Alzheimer's disease and Other Cognitive Disorders Unit, Neurology Service, Hospital Clínic, Barcelona, Spain;

- Jessica Panman - Department of Neurology, Erasmus Medical Center, Rotterdam, Netherlands;

- Janne Papma - Department of Neurology, Erasmus Medical Center, Rotterdam;

- Michela Pievani - Istituto di Ricovero e Cura a Carattere Scientifico Istituto Centro San Giovanni di Dio Fatebenefratelli, Brescia, Italy;

- Yolande Pijnenburg -Amsterdam University Medical Centre, Amsterdam VUmc, Amsterdam, Netherlands;

- Cristina Polito - Department of Biomedical, Experimental and Clinical Sciences "Mario Serio", Nuclear Medicine Unit, University of Florence, Florence, Italy

- Sara Prioni -Fondazione IRCCS Istituto Neurologico Carlo Besta, Milano, Italy;

- Catharina Prix -Neurologische Klinik, Ludwig-Maximilians-Universität München, Munich, Germany; 
- Rosa Rademakers [as London Ontario geneticist] - Department of Neurosciences, Mayo Clinic, Jacksonville, Florida, USA;

- Veronica Redaelli -Fondazione IRCCS Istituto Neurologico Carlo Besta, Milano, Italy;

- Tim Rittman - Department of Clinical Neurosciences, University of Cambridge, Cambridge, UK;

- Ekaterina Rogaeva -Tanz Centre for Research in Neurodegenerative Diseases, University of Toronto, Toronto, Canada;

- Pedro Rosa-Neto -Translational Neuroimaging Laboratory, McGill Centre for Studies in Aging, McGill University, Montreal, Québec, Canada;

- Giacomina Rossi - Fondazione IRCCS Istituto Neurologico Carlo Besta, Milano, Italy;

- Martin Rossor - Dementia Research Centre, Department of Neurodegenerative Disease, UCL Institute of Neurology, Queen Square, London, UK;

- Beatriz Santiago - Neurology Department, Centro Hospitalar e Universitario de Coimbra, Coimbra, Portugal;

- Elio Scarpini -Fondazione IRCCS Ca' Granda Ospedale Maggiore Policlinico, Neurodegenerative Diseases Unit, Milan, Italy; University of Milan, Centro Dino Ferrari, Milan, Italy;

- Sonja Schönecker - Neurologische Klinik, Ludwig-Maximilians-Universität München, Munich, Germany;

- Elisa Semler -Department of Neurology, University of Ulm, Ulm;

- Rachelle Shafei - Dementia Research Centre, Department of Neurodegenerative Disease, UCL Institute of Neurology, Queen Square, London, UK;

- Christen Shoesmith - Department of Clinical Neurological Sciences, University of Western Ontario, London, Ontario, Canada;

- Miguel Tábuas-Pereira - Neurology Department, Centro Hospitalar e Universitario de Coimbra, Coimbra, Portugal;

- Mikel Tainta - Neuroscience Area, Biodonostia Health Research Insitute, San Sebastian, Gipuzkoa, Spain;

- Ricardo Taipa - Neuropathology Unit and Department of Neurology, Centro Hospitalar do Porto - Hospital de Santo António, Oporto, Portugal;

- David Tang-Wai -The University Health Network, Krembil Research Institute, Toronto, Canada;

- David L Thomas - Neuroimaging Analysis Centre, Department of Brain Repair and Rehabilitation, UCL Institute of Neurology, Queen Square, London, UK;

- Paul Thompson - Division of Neuroscience and Experimental Psychology Wolfson Molecular Imaging Centre, University of Manchester, Manchester, UK;

- Hakan Thonberg- Center for Alzheimer Research, Division of Neurogeriatrics, Karolinska Institutet, Stockholm, Sweden;

- Carolyn Timberlake- Department of Clinical Neurosciences, University of Cambridge, Cambridge, UK;

- Pietro Tiraboschi - Fondazione IRCCS Istituto Neurologico Carlo Besta, Milano, Italy;

- Philip Van Damme - Neurology Service, University Hospitals Leuven, Belgium; Laboratory for Neurobiology, VIB-KU Leuven Centre for Brain Research, Leuven, Belgium;

- Mathieu Vandenbulcke - Geriatric Psychiatry Service, University Hospitals Leuven, Belgium; Neuropsychiatry, Department of Neurosciences, KU Leuven, Leuven, Belgium;

- Michele Veldsman - Nuffield Department of Clinical Neurosciences, Medical Sciences Division, University of Oxford, Oxford, UK;

- Ana Verdelho - Department of Neurosciences and Mental Health, Centro Hospitalar Lisboa Norte - Hospital de Santa Maria \& Faculty of Medicine, University of Lisbon, Lisbon, Portugal;

- Jorge Villanua - OSATEK, University of Donostia, San Sebastian, Gipuzkoa, Spain;

- Jason Warren - Dementia Research Centre, Department of Neurodegenerative Disease, UCL Institute of Neurology, Queen Square, London, UK;

- Carlo Wilke -Department of Neurodegenerative Diseases, Hertie-Institute for Clinical Brain Research and Center of Neurology, University of Tübingen, Tübingen, Germany; Center for Neurodegenerative Diseases (DZNE), Tübingen, Germany;

- Ione Woollacott - Dementia Research Centre, Department of Neurodegenerative Disease, UCL Institute of Neurology, Queen Square, London, UK;

- Elisabeth Wlasich - Neurologische Klinik, Ludwig-Maximilians-Universität München, Munich, Germany;

- Henrik Zetterberg - Dementia Research Institute, Department of Neurodegenerative Disease, UCL Institute of Neurology, Queen Square, London, UK;

- Miren Zulaica - Neuroscience Area, Biodonostia Health Research Insitute, San Sebastian, Gipuzkoa, Spain; 